\title{
Major Depressive Disorder and the "Leaky Gut” Post Cholecystectomy
}

\author{
Sibin Nair ${ }^{1}$ and Ossama Riaz ${ }^{2 *}$ \\ 1Saint James School of Medicine, Saint Lucia \\ ${ }^{2}$ Spartan Health Sciences University, Saint Lucia
}

*Corresponding author: Ossama Riaz, Spartan Health Sciences University, Saint

Case Report
Volume 2 Issue 6
Received Date: October 04,2018
Published Date: November 26,2018
DOI: $10.23880 /$ mjccs-16000187

Lucia; Email: ossamariaz@gmail.com

\section{Summary}

Major Depressive Disorder (MDD) is the most common mental health disorder in the United States as well as all over the world. According to CDC, MDD affects more than 16.1 million American adults, or about 6.7\% of the U.S. population age 18 and older in a given year. People suffering from MDD experience sadness, anhedonia, feelings of guilt, loss of energy, lack of concentration, loss in appetite, psychomotor symptoms with possible suicidal ideation. It is a mental disease that is still marginalized and ostracized in society today. We wrote this case report in hopes to raise awareness of depression that can affect individuals through secondary pathways.

Key words: Cholecystectomy; Anhedonia; Hypocalcemia; Malabsorption; Lipophilic

Abbreviations: MDD: Major Depressive Disorder; SIBO: Small Intestinal Bacterial Overgrowth; BDNF: BrainDerived Neurotrophic Factor.

\section{Background}

Gastric acid is involved in the primary function of preparing food for digestion and absorption by the intestine. Acid production is a central component of the stomach's contribution to digestion. Low gastric acid means higher susceptibility to food poisoning. Proton pump inhibitors can cause diarrhea, enteric infections, and alter the gastrointestinal bacterial population by suppressing the gastric acid barrier leading to small intestinal bacterial overgrowth (SIBO). Normally when food leaves the stomach, it is acidic, and it leads to changes in $\mathrm{pH}$ and the pancreas and gallbladder release pancreatic juice and bile but if the stomach is not producing gastric acid, the gallbladder stops releasing bile. Prolonged PPI treatment produces bowel symptoms and symptoms like cramps, hypotension and hypomagnesemia. The gallbladder has a very important role to play as the same nutrients that work in the gallbladder produces bile. Therefore, when a patient undergoes cholecystectomy, they develop an imbalance of neurotransmitters. Bile makes glycine and taurine and when it is depleted we cannot absorb calcium and this leads to hypocalcemia. Gallbladder bile rinses bacteria off the wall of our gut and the bacteria sticks to the wall, rising in the bowel leading to gastrointestinal symptoms such as malabsorption. The bacteria are now rising, and it is considered the "leaky gut" bacteria causing inflammation and depression. Increased intestinal permeability associated with an upload of LPS 


\section{Medical Journal of Clinical Trials \& Case Studies}

translocation induces depression symptoms. Gut microbiota and probiotics alter behavior and brain neurochemistry.

\section{Case Presentation}

In this case, our patient is a 62-year-old female who underwent cholecystectomy after feeling months of gastric discomfort and dyspepsia. Her ultrasound imaging and lab results pointed to a diagnosis of cholecystitis and she presented to the ER with intractable abdominal pain. After initial stabilization and pain management, the patient in agreement with her GI doctor, decided to have a cholecystectomy performed as she had been struggling with cholecystitis for years now. The procedure was done laparoscopically with no post operational complications. Three months later the patient presented with severe fatigue and weight gain. She did not feel like leaving her house, stopped socializing with her friends, felt a loss of energy and just seemed to stop caring about things. She was taken to her PCP who recommended a psychiatry consult where she was diagnosed with moderate depression. The purpose of this report is to theorize how her enteric system undergoing chronic inflammation along with her cholecystectomy further exacerbated the inflammation and ultimately leading to depression. This case report will delve into the "Leaky Gut" Phenomenon and how it led to the development of her depression.

\section{Past Medical History}

Hyperlipidemia, Gastroesophageal Reflux Disease, Hypertension, Gastritis, and obesity.

\section{Past Family History}

The patient's father and mother both have a history of hypertension, gastroesophageal reflux Disease and hyperlipidemia. Both parents now deceased, died of cardiac causes.

\section{Past Social History}

Patient denies use of alcohol, nicotine and illegal drugs.

\section{Differential Diagnosis}

- Social Anxiety

- Gastroesophageal Reflux Disease

- Post-cholecystectomy Syndrome

- Cholecystitis

- Cholelithiasis

- Factitious Disorder
- Munchausen Syndrome

- Major Depression Disorder

- Generalized Anxiety Disorder

- Panic Disorder

- Primary Hypertension

- Secondary Hypertension

\section{Treatment}

The patient besides the pain of surgery felt well postsurgery and had hoped her symptoms of abdominal pain, continuous GERD, IBS, and nausea would get better. She was prescribed cholestyramine and esomeprazole to deal with any residual symptoms. The patient was also scheduled to follow up with a dietician who prescribed a course of medical nutrition therapy individualized to our patient. The patient was advised to limit fat intake, increase dietary fiber gradually, and to avoid caffeinated and carbonated drinks, chocolate, citrus products, spicy foods and to eat small meals. Due to a combination of factors including non-compliance with proposed dietary restrictions and a return of pre-surgical symptoms, our patient started experiencing depression and a complete loss of interest in activities of daily living and was diagnosed with moderate depression by a psychiatrist. The psychiatrist after a careful review of the patient's file suggested a thorough course of cognitive behavioral therapy, a modified and realistic diet in consultation with a dietician, a workout routine and therapy sessions relating to the patient's depression and eating habits. After two months of little improvement, the patient was started on Wellbutrin by her psychiatrist keeping the associated weight gain with other antidepressants in mind.

\section{Outcome and Follow Up}

Although our patient was given the appropriate postop care she initially felt better but then began to deteriorate to her pre-surgery levels in terms of symptoms and now has new feelings of depression which could have been related to her non-compliance with her recommendations in terms of her diet and other appropriate lifestyle changes. After a psychiatric consult and initial assessment, the patient tried to be more adherent to the recommendations and hoped her CBT sessions, therapy, and new dietary guidelines coupled with exercise would alleviate her concerns yet she continued to feel depressed and continued to have abdominal symptoms similar to what she felt before she had her cholecystectomy. The patient was then prescribed 


\section{Medical Journal of Clinical Trials \& Case Studies}

Wellbutrin and advised to continue her CBT and follow her dietary, exercise, and healthy lifestyle recommendations. After an intense and strict adherence to her diet/exercise/CBT routine together with the medication the patient experienced mild relief within five months post-surgery, her abdominal symptoms had disappeared, and she had started to lose weight and felt happier. The patient started to socialize more and took up a proactive approach to bettering her eating habits and other lifestyle choices. The Wellbutrin initially started at $200 \mathrm{mg}$ twice a day was maintained as the patient did not feel like there was any need for an adjustment.

\section{Discussion}

A dysbiotic state leads to increased intestinal permeability and allows contents such as bacterial metabolites and molecules as well as bacteria themselves to leak through the submucosa and into the systemic circulation, a phenomenon aptly named leaky gut syndrome [1]. In our digestive gut, sugar precursors E. coli \& proteobacteria Bifidobacterium species act on the chorismite from where E Coli, proteobacteria, B. subtilis, firmevlves, mycobacterium and actinobacteria leads to formation of tryptophan which in the intestinal epithelial cells causes serotonin production in the gut. When the concentration of these bacteria is reduced due to antibiotics, it decreases their quantity in the gut and the lack of bile cleanses the gut lining and gets rid of the bad bacteria. It leads to less tryptophan and serotonin being produced leading to depression. Tryptophan is synthesized from chorismite by members of several bacterial phyla including protein bacterium, actinobacterium \& fircules. E Coli can synthesize chorismite, a tryptophan precursor, which acts as a branch point for microbial metabolic pathways consistent with this key role for bacteria. Antibiotics treatment alters tryptophan and undoes metabolism leading to less serotonin and melatonin in the brain. Tryptophan, tyrosine, and phenylalanine made in gut by bacteria. When tryptophan is shuttled through the kynurenine pathway there is less tryptophan available to make serotonin and hence less serotonin in the gut available to be transported to the brain via the vagal nerve. Low bile post-cholecystectomy causes leaky gut as bile acid stimulates biliary lipid secretion due to their physical chemical properties. They can form mixed micelles together with biliary phospholipids which allows the solubilizing in bile of cholesterol and other lipophilic compounds. Mixed micelles also account for the emulsion of dietary fat and lip soluble vitamins in the gut, helping their absorption. Bile acids also facilitate intestinal calcium absorption. At the intestinal level, bile acids are known to modulate pancreatic enzyme secretion and cholecystokinin release. Moreover, they are potent antimicrobial agents that prevent bacterial over growth in the small bowel.

There is also a systemic IgM-mediated immune response in depression directed against LPS (which is part of the bacterial wall of gram negative bacteria) suggesting that bacterial translocation may play a role in the inflammatory and pathophysiology of clinical depression. Bacterial translocation indicates the presence of "leaky gut" or an increased permeability of the gut wall or loosening of the tight junction barrier [2]. Hyperactivity of the HPA axis is also one of the most reliable biological findings in patients suffering from major depression. Exposure to stress and cytokine responses may impair neuronal plasticity and stimulation of neurotransmission. Administration of exogenous IL1 bsignificantly enhanced IL-1b levels in the PFC and hippocampus and further increased HPA axis activity. Likewise, acute stress markedly increased IL-1b levels in the PFC, hippocampus and hypothalamus. Repeated stress sensitized the HPA axis response to administration of exogenous IL-1band possibly leads to depression [3].

The gut is composed of nerve tissues and uses neurotransmitters to communicate with the brain. The gut microbiota affects brain development and plasticity by secreting various neurotrophins and proteins, such as brain-derived neurotrophic factor (BDNF), synaptophysin and postsynaptic density [4]. Our brain has an intimate connection with our gut via vagal nerve, which has hundreds of millions of nerves cells that regulate our digestive processes. This "second brain" in the gut is spread out throughout the entire length of the gut. This second brain has many sensors in the enteric nervous system. The connection between depression and the gut is integrative from serotonin in the gut and how it relates to serotonin in the brain. Many patients with depression complain of constipation. This might be due to $95 \%$ of our serotonin being produced and stored in the gut via enterochromaffin cells which leads to modulation of mood, appetite, and well-being. The serotonin in the gut is synthesized from the food that we inject and the microbes that live in our gut that stimulate the production of serotonin. $60 \%$ of the production is due to the signals that come from the microbes in our gut. The serotonin cells are sandwiched between the cells that make the lining of the gut. The cells have vagal nerve association and the 


\section{Medical Journal of Clinical Trials \& Case Studies}

signal when the cell is activated goes to the brain via the vagal deliver into the limbic system. This case study involves a patient with a long history of GERD treated with $\mathrm{H} 2$ antagonists who underwent cholecystectomy. This was followed by symptoms of depression that required treatment with an ant-depressant. This case study is being written to establish a possible connection between the gut and depression.

\section{References}

1. Clapp M, Aurora N, Herrera L, Bhatia M, Wilen E, et al. (2017) Gut microbiota's effect on mental health: The gut-brain axis. Clin Pract 7(4): 987.
2. Berk M, Williams LJ, Jacka FN, Adrienne O'Neil, Julie A Pasco, et al. (2013) So depression is an inflammatory disease, but where does the inflammation come from? BMC Med 11: 200.

3. Gądek-Michalska A, Tadeusz J, Rachwalska P, Bugajski J (2013) Cytokines, prostaglandins and nitric oxide in the regulation of stress-response systems. Pharmacol Rep 65(6): 1655-1662.

4. Evrensel A, Ceylan ME (2015) The Gut-Brain Axis: The Missing Link in Depression. Clin Psychopharmacol Neurosci 3(3): 239-244. 